\title{
Relativistic density functional for nuclear matter
}

\author{
Stefan Gmuca $^{1, *}$, Kristian Petrík ${ }^{1}$, and Jozef Leja ${ }^{1}$ \\ ${ }^{1}$ Institute of Physics, Slovak Academy of Sciences, Dubravska cesta 9, Bratislava, Slovakia
}

\begin{abstract}
In the present work, we have mapped the exchange Fock contributions from the Dirac-Hartree-Fock (DHF) approach for nuclear matter onto the direct Hartree terms. This results in the relativistic mean field (RMF) model with the density dependent couplings. The density dependence of the effective coupling constants thus reflects the exchange correlations. The exchange part of an energy density of the linear DHF model in dense matter is evaluated in a parameter-free closed form and, after the rearrangement of the terms, expressed as density functional.
\end{abstract}

\section{Introduction}

One of the central issues of current nuclear physics is to develop the density functional for a proper description of exotic nuclei and equation-of-state (EOS) of nuclear matter. The relativistic mean field (RMF) theory $[1,2]$ seems to be a suitable starting candidate for this task. The RMF model has greatly contributed to our understanding of nuclear structure, and it was intensively used over years for a description of a wide class of nuclear phenomena (see e.g. [3] and references therein for recent reviews). Two versions of the RMF approach have been developed, either one with nonlinear self-interactions of meson fields [4], or one with density dependent meson-nucleon couplings [5]. The approaches introduce the density dependence into the effective interaction. That is inevitable for a proper description of nuclear systems.

In addition, there exist approaches incorporating the exchange (Fock) terms into the relativistic description of nuclear matter and finite nuclei. The relativistic Dirac-Hartree-Fock (DHF) approach [6] has been developed together with its own density dependent versions [7]. The exchange parts give rise to the state-dependent potentials due to nonlocal character of the DHF approach and the solutions of Dirac equations for finite nuclei thus require much more numerically intensive efforts than in the RMF model.

It is, therefore, of interest to develop an approach as simple as the RMF model that will account for exchange correlations, at least for nuclear matter [8].

The density dependent couplings in the RMF and DHF approaches are introduced phenomenologically. They are often determined by comparison with Dirac-Brueckner-HartreeFock (DBHF) calculations for nuclear matter [5] in limited range of densities. Their functional forms are usually chosen $a d-h o c$, the choice being dictated mainly by the requirement of simplicity. Most authors employ the rational form. Various functions of density dependences used are discussed in Ref. [9] together with their extrapolation properties out of fitted interval of densities. Especially, the couplings for low density region (relevant for nuclear

\footnotetext{
*e-mail: gmuca@savba.sk
} 
periphery of exotic nuclei) and high density one (typical for neutron star calculations) are quite unreliably determined by extrapolation.

This contribution is aimed at mapping of the Fock exchange terms of the DHF approach for nuclear matter onto its Hartree parts. An explicit evaluation of the exchange integrals for self-energy components leads to the RMF theory with density dependent couplings. The analytical functional density dependence of couplings obtained thus accounts for exchange correlations over full range of densities. This may significantly reduce the arbitrariness of couplings at low and high densities.

For simplicity we will treat the symmetric nuclear matter case and consider the exchange of isoscalar mesons only, together with $\pi$-meson. While the former give the dominant contributions in both, the RMF and DHF approaches, the pionic field contributes only in exchange part of the DHF model. This illustrates the essential points of the approach. The generalization for asymmetric nuclear matter and full set of exchanged mesons can be made straightforwardly.

\section{Relativistic Hartree-Fock for nuclear matter}

Here we present the main ingredients of the relativistic (Dirac) Hartree-Fock (DHF) approach for the symmetric nuclear matter [6]. For simplicity we start with the effective Lagrangian density for the interacting nucleons $(\psi)$ and the scalar $\sigma$, vector $\omega$, and pion $\pi$ fields only,

$$
\mathcal{L}=\mathcal{L}_{0}+\mathcal{L}_{I}
$$

consisting of the free parts

$$
\begin{aligned}
\mathcal{L}_{0}= & \bar{\psi}\left(i \gamma_{\mu} \partial^{\mu}-M\right) \psi+\frac{1}{2}\left(\partial_{\mu} \sigma \partial^{\mu} \sigma-m_{\sigma}^{2} \sigma^{2}\right) \\
& -\frac{1}{4} \omega_{\mu \nu} \omega^{\mu \nu}+\frac{1}{2} m_{\omega}^{2} \omega_{\mu} \omega^{\mu}+\frac{1}{2}\left(\partial_{\mu} \pi \partial^{\mu} \pi-m_{\pi}^{2} \pi^{2}\right),
\end{aligned}
$$

where $\omega_{\mu \nu}=\partial_{\mu} \omega_{v}-\partial_{\nu} \omega_{\mu}$, and the interaction terms

$$
\mathcal{L}_{I}=-g_{\sigma} \bar{\psi} \sigma \psi-g_{\omega} \bar{\psi} \gamma_{\mu} \omega^{\mu} \psi-\frac{f_{\pi}}{m_{\pi}} \bar{\psi} \gamma_{5} \gamma_{\mu}\left(\partial^{\mu} \pi\right) \psi
$$

Here, the symbol $M$ denotes the nucleon rest mass, whereas $g_{i}\left(f_{i}\right), m_{i}, i=\{\sigma, \omega, \pi\}$ mean the coupling constants and rest masses for the scalar $(\sigma)$, vector $(\omega)$ and pseudoscalar $(\pi)$ mesons, respectively. The direct Yukawa couplings are used for the $\sigma$ and $\omega$ fields, while the pseudovector coupling is applied for the pion.

Due to parity and time reversal symmetry, the nuclear matter self-energy $\Sigma$ takes a form

$$
\Sigma(k)=\Sigma^{s}(k)-\gamma^{0} \Sigma^{0}(k)+\boldsymbol{\gamma} \cdot \boldsymbol{k} \Sigma^{v}(k),
$$

where superscripts $\{s, 0, v\}$ denote the scalar, time-like and space-like components of the self-energy, consecutively.

Using the standard techniques of the DHF approach, the components of the nucleon selfenergy in nuclear matter at Fermi momentum $k_{F}$ can be finally written as 


$$
\begin{aligned}
\Sigma^{s}\left(k_{F}, k\right)= & -\frac{\gamma}{(2 \pi)^{3}} \frac{g_{\sigma}^{2}}{m_{\sigma}^{2}} \int_{0}^{k_{F}} d^{3} q \frac{M^{\star}(q)}{E^{\star}(q)}+\frac{1}{(4 \pi)^{2} k} \int_{0}^{k_{F}} q d q \frac{M^{\star}(q)}{E^{\star}(q)} \\
& {\left[g_{\sigma}^{2} \Theta_{\sigma}(k, q)-4 g_{\omega}^{2} \Theta_{\omega}(k, q)-3\left(\frac{f_{\pi}}{m_{\pi}}\right)^{2} m_{\pi}^{2} \Theta_{\pi}(k, q)\right], } \\
\Sigma^{0}\left(k_{F}, k\right)= & -\frac{\gamma}{(2 \pi)^{3}} \frac{g_{\omega}^{2}}{m_{\omega}^{2}} \int_{0}^{k_{F}} d^{3} q-\frac{1}{(4 \pi)^{2} k} \int_{0}^{k_{F}} q d q \\
& {\left[g_{\sigma}^{2} \Theta_{\sigma}(k, q)+2 g_{\omega}^{2} \Theta_{\omega}(k, q)-3\left(\frac{f_{\pi}}{m_{\pi}}\right)^{2} m_{\pi}^{2} \Theta_{\pi}(k, q)\right], }
\end{aligned}
$$

where

$$
\Theta_{i}(k, q)=\ln \frac{(k+q)^{2}+m_{i}^{2}}{(k-q)^{2}+m_{i}^{2}},
$$

and

$$
\Phi_{i}(k, q)=\frac{1}{4 k q}\left(k^{2}+q^{2}+m_{i}^{2}\right) \Theta_{i}(k, q)-1
$$

are angular exchange integrals and $\gamma$ is a spin-isospin degeneracy factor ( $\gamma=4$ for symmetric nuclear matter). Other symbols used have their usual meaning. Here we already omitted the retardation effects in the meson propagators and subtracted the zero-range contribution from the pion exchange.

The first term in $\Sigma^{s}\left(k_{F}, k\right)$ and $\Sigma^{0}\left(k_{F}, k\right)$ corresponds to the Hartree contribution and may be related to scalar and baryon densities according to the following expressions,

$$
\rho_{s}\left(k_{F}\right)=\frac{\gamma}{(2 \pi)^{3}} \int_{0}^{k_{F}} d^{3} q \frac{M^{\star}(q)}{E^{\star}(q)}
$$

and

$$
\rho_{B}\left(k_{F}\right)=\frac{\gamma}{(2 \pi)^{3}} \int_{0}^{k_{F}} d^{3} q=\frac{2}{3 \pi^{2}} k_{F}^{3},
$$

respectively. The remaining terms are due to the exchange (Fock) contributions.

The Eqs. (5)-(6) are the integral equations for the individual components of the nuclear matter self-energy and they have to be solved self-consistently together with expressions (9) and (10).

\section{Effective couplings}

In the next step we determine the effective density dependent coupling constants for the scalar and vector mesons by an explicit evaluation of Fock expressions and relating them to the Hartree parts through the effective couplings. The procedure to be demonstrated for the vector meson coupling.

By inspecting the Fock integral in Eq. (6) one reveals that each particular contribution depends on the exchange integral of the type

$$
I_{e x c}\left(k_{F}, k\right)=\frac{1}{k} \int_{0}^{k_{F}} q d q \ln \frac{(k+q)^{2}+m^{2}}{(k-q)^{2}+m^{2}} .
$$

The integral may be evaluated in the closed expression and exhibits a weak, almost parabolic dependence on momentum $k$ in the interval from zero up to $k_{F}$. This is illustrated 


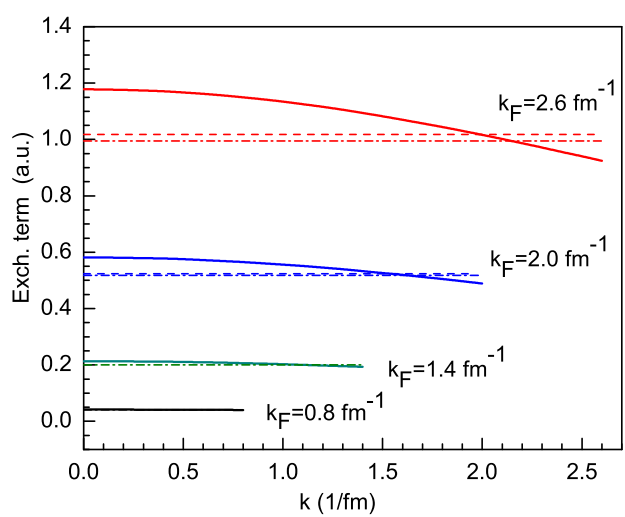

Figure 1. (Color online.) The relative momentum dependence of the exchange integral (solid lines) at various Fermi momenta (see text). The dashdotted lines represent the $k$-averaged values according Eq. (13), while dashed lines come from averaging of full exact calculations. The results presented are for $m=4 \mathrm{fm}^{-1}$

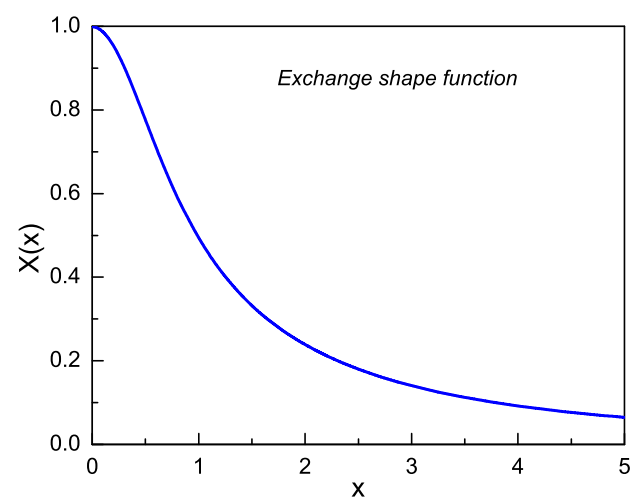

Figure 2. (Color online.) The plot of auxiliary exchange shape function $X(x)$ (see Eq. (16))

in Fig. 1 where the Fermi momenta chosen cover a wide range of densities up to a few times the saturation density.

The momentum dependence of the exchange terms (and thus self-energy) is the genuine feature of the relativistic Hartree-Fock approach. To simplify model and compare with the $\mathrm{RMF}$ one this $k$-dependence has to be integrated out. Usually, the exchange parts are either evaluated at the $k=k_{F}$ momentum, or are $k$-averaged. Both approaches, however, lead to rather cumbersome expressions difficult to manipulate with.

In the present paper we adopted a modified approach. Instead, we

(a) evaluated the exchange integral,

(b) expanded it in $k$ at $k=0$ and kept the terms up to $k^{2}, O\left(k^{4}\right)$,

(c) averaged it over the Fermi sphere.

Thus, the $k$-averaged exchange integral may be given by a pretty compact and simple closed expression, that reads

$$
\bar{I}_{e x c}\left(k_{F}\right)=4\left(k_{F}-m \arctan \left(\frac{k_{F}}{m}\right)-\frac{k_{F}^{5}}{5\left(k_{F}^{2}+m^{2}\right)^{2}}\right) .
$$

Here, the first two terms come from the evaluation of exchange integral at $k=0$ and the third term is due to $k$-averaging procedure. The difference between the exact numerically averaged results for the exchange integral and that given by Eq. (13) is less than $2 \%$ even for highest Fermi momenta considered (as it may be seen in Fig. 1).

This result allows us to evaluate all the exchange terms of the vector component of nuclear matter self-energy in Eq. (6). Finally, we may rewrite Eq. (6) as,

$$
\Sigma^{0}\left(k_{F}\right)=\frac{\Gamma_{\omega}^{2}\left(k_{F}\right)}{m_{\omega}^{2}} \rho_{B}\left(k_{F}\right) .
$$


This way, we are projecting the vector component of the DHF self-energy onto mean-field level, now, however, with the effective density-dependent (in fact, Fermi momentum dependent) meson-nucleon coupling vertex $\Gamma_{\omega}^{2}\left(k_{F}\right)$. It reads

$$
\frac{\Gamma_{\omega}^{2}\left(k_{F}\right)}{m_{\omega}^{2}}=\frac{g_{\omega}^{2}}{m_{\omega}^{2}}+\sum_{i=\{\sigma, \omega, \pi\}} a_{i} \frac{g_{i}^{2}}{m_{i}^{2}} X\left(k_{F} / m_{i}\right),
$$

where we have introduced the auxiliary function

$$
X(x)=3\left(x-\arctan (x)-\frac{x^{5}}{5\left(1+x^{2}\right)^{2}}\right) / x^{3},
$$

normalized as $X(0)=1$, and the coefficients $a_{i}$ are listed in Tab. 1. The function $X(x)$ is plotted in Fig. 2 for a wide range of the dimensionless variable $x=k_{F} / m$. We note that for all mesons except the lightest one $\pi$, the variable $x$ takes values less than 1 in most of cases.

Table 1. The numerical fractions $a_{i}$ and $b_{i}$

\begin{tabular}{rrrr}
\hline$i$ & $\sigma$ & $\omega$ & $\pi$ \\
\hline$a_{i}$ & $+\frac{1}{8}$ & $+\frac{2}{8}$ & $-\frac{3}{8}$ \\
$b_{i}$ & $-\frac{1}{8}$ & $+\frac{4}{8}$ & $+\frac{3}{8}$
\end{tabular}

Analogically, for the scalar effective density dependent coupling we obtain the relation

$$
\frac{\Gamma_{\sigma}^{2}\left(k_{F}\right)}{m_{\sigma}^{2}}=\frac{g_{\sigma}^{2}}{m_{\sigma}^{2}}+\sum_{i=\{\sigma, \omega, \pi\}} b_{i} \frac{g_{i}^{2}}{m_{i}^{2}} X\left(k_{F} / m_{i}\right) .
$$

The results obtained represents the mean field model with the density dependent coupling constants at the Hartree level that accounts for the exchange correlations. The approach is parameter-free and may be easily cast on full range of exchanged mesons, as well as to consider wider set of baryons. In addition, the asymmetric composition of nuclear matter may be taken into account.

\section{Summary}

In the present work we have mapped the exchange Fock contributions from the DHF approach for nuclear matter onto the direct Hartree terms. This results in the relativistic mean field model with the density dependent couplings. The density dependence of the effective coupling constants reflects the exchange correlations. While this work is restricted to the exchange of scalar and vector mesons only, the approach itself can be straightforwardly generalized for the full set of mesons, and for asymmetric nuclear matter. Contrary to the relativistic density dependent hadron field theory, which uses the phenomenological density dependence of coupling constants, the present approach introduces no additional free parameters into the model. Thus, this contribution is a step in a direction to formulate the effective nuclear density functional suitable for description of wide class of nuclear phenomena over a broad range of densities. Such a development is of utmost importance since the theory is 
needed able to predict nuclear properties in regions of the nuclear chart not yet accessible to experiment. Now it is believed that the relativistic self-consistent mean field model with the density dependent couplings is a good approach to reach this goal.

Acknowledgements. This work was supported in part by the VEGA Grant Agency under the project No. 2/0176/16 and by the Slovak Research and Development Agency under the contract No. APVV-15-0225.

\section{References}

[1] L.D. Miller and A.E.S. Green, Phys. Rev. C 5, 241 (1972)

[2] J.D. Walecka, Ann. Phys. (N.Y.) 83, 491 (1974)

[3] M. Bender, P.-H. Heenen and P.-G. Reinhard, Rev. Mod. Phys. 75, 121 (2003)

[4] J. Boguta and A. R. Bodmer, Nucl. Phys. A 292, 413 (1977). A. R. Bodmer, Nucl. Phys. A 526, 703 (1991). S. Gmuca, Nucl. Phys. A 547, 447 (1992). H. Mueller and B. D. Serot, Nucl. Phys. A 606, 508 (1996)

[5] C. Fuchs, H. Lenske, and H.H. Wolter, Phys. Rev. C 52, 3043 (1995). H. Shen, Y. Sugahara, and H. Toki, Phys. Rev. C 55, 1211 (1997). S. Typel and H.H. Wolter, Nucl. Phys. A 656, 331 (1999). F. Hofmann, C. M. Keil and H. Lenske, Phys. Rev. C 64, $034314(2001)$

[6] C. J. Horowitz and B. D. Serot, Phys. Lett. 109B, 341 (1982); Nucl. Phys. A 399, 529 (1983), A. Bouyssy, S. Marcos, J.-F. Mathiot, and N. Van Giai, Phys. Rev. Lett. 55, 1731 (1985). A.Bouyssy, J.-F. Mathiot, N. Van Giai, and S. Marcos, Phys. Rev. C 36, 380 (1987)

[7] R. Fritz, H. Müther, and R. Machleidt, Phys. Rev. Lett. 71, 46 (1993). Hua-lin Shi, Bao-qiu Chen, and Zhong-yu Ma, Phys. Rev. C 52, 144 (1995)

[8] N. Van Giai et al., J. Phys. G: Nucl. Part. Phys. 37, 064043 (2010)

[9] K. Petrík and S. Gmuca, J. Phys. G: Nucl. Part. Phys. 39, 085113 (2012) 